\title{
Distributed Power Control and Routing for Clustered CDMA Wireless Ad Hoc Networks
}

\author{
Aylin Yener \\ Electrical Engineering Department \\ The Pennsylvania State University \\ University Park, PA 16802 \\ yener@ee.psu.edu
}

\author{
Shalinee Kishore \\ Department of Electrical and Computer Engineering \\ Lehigh University \\ Bethlehem, PA 18015 \\ skishore@lehigh.edu
}

\begin{abstract}
We consider a multi-hop wireless ad hoc network where all nodes share a common channel, and Code Division Multiple Access (CDMA) is used to facilitate concurrent transmissions. The network model consists of groups of nodes that may loosely be termed as clusters. We address the question of how to efficiently facilitate intra- and inter-cluster communications between multiple source-destination pairs. The performance metric considered is throughput per unit power. The concurrent transmissions employ distributed power control and are scheduled to resolve conflicts. We propose a dynamic forwarding mechanism in which forwarding nodes are used when it is energy-efficient, and the choice of forwarding node varies according to sourcedestination pairs. We observe that such a dynamic forwarding policy yields improved throughput per unit power as compared to previously proposed schemes which use either fixed forwarding nodes or cluster-heads to perform inter-cluster communications.
\end{abstract}

\section{INTRODUCTION}

Ad hoc networks are becoming increasingly popular in enabling high speed wireless communications, their most obvious advantage being the lack of infrastructure requirement and hence flexible and rapid deployment. The design of a wireless ad hoc network, however, entails additional challenges as compared to cellular wireless communications systems. In particular, design solutions not only have to deal with the radio channel and provide sufficient capacity over limited bandwidth resources, but the absence of centralized control units, i.e., base stations, dictate that these solutions be distributed in nature. It is now widely agreed that the wireless ad hoc network design greatly benefits from jointly designing several layers of the network allowing the interaction between the physical, link, medium access and network layers [1], [2].

We note that recently intense research efforts have addressed several issues pertaining to wireless ad hoc network design at several layers. For example, while earlier efforts in ad hoc network deployment assumed one node transmits at a time, e.g., $802.11 \mathrm{x}$, recent work has addressed the possibility of concurrent transmissions and the use of CDMA [3], [4]. Power control has also been considered for ad hoc networks [5]. References [6]-[8] determine the minimum power levels needed to reach the destination in ad hoc networks with nonconcurrent transmissions, which helps control the range in which a node can transmit. On the other hand, references [4], [9] consider implementation of power control when concurrent transmissions are allowed. In this case, power control serves not only to limit the transmission range, but also as an interference management tool which enables multiple simultaneous successful transmissions that are in transmission range of each other.

The lack of coordinating nodes in an ad hoc network can create significant design challenges. Sizeable research effort has been directed towards introducing a class of network nodes that would perform coordination functions, such as transmission coordination and resource allocation. In such cases, a group of local nodes would be served by a basestation-like entity called the cluster head [10]. Such grouping of nodes, often easily justified via the physical proximity of the nodes, greatly eases the link layer and routing design, since node communicate to each other via the cluster heads. Implementation of power control also becomes easier with the availability of power control feedback from the cluster head [4], [11].

In this paper, we attempt to jointly address several of the issues encountered in developing distributed resource allocation solutions for wireless multi-hop ad hoc networks. We consider ad hoc networks where the node distribution is non-uniform, giving rise to several physically close groups of nodes, i.e., clusters. Our aim is to come up with solutions that will enhance the throughput of the overall network per unit power expended, that is to come up with an energy efficient network design [4], [12], [13]. In doing so, we must consider the issues that arise related to physical, link and network layers.

We consider a shared channel and allow concurrent transmissions of nodes via the employment of CDMA as the multiple access scheme. Interference management for concurrently transmitting nodes is achieved by distributed power control. Routing decisions from each source node to each destination node are made to achieve better throughput per power. In particular, we motivate and show that energy efficiency is improved when dynamically assigned forwarding nodes are utilized for inter-cluster communications instead of fixed nodes. When implementing these routes, medium access is provided if all transmitting nodes can be received reliably, i.e., at their quality of service (QoS) target. If this is not feasible, then some transmissions are delayed. In addition, we schedule transmissions to avoid simultaneous transmit/receive 
conflicts. The notion that transmissions occur according to such prescribed schedules is used throughout this paper. These schedules, however, are not necessarily determined by a centralized entity but can be seen as the transmission sequences that result after distributed random access.

The choice of forwarding nodes for the active source destination pairs provides a list of required transmissions. This transmission list requires scheduling to avoid simultaneous transmission and reception at each node. In addition, the schedule ensures only feasible simultaneous transmissions occur at any given time, i.e., transmitting nodes are able to achieve their QoS targets. Hence routing and medium access are tightly coupled and they in turn impact interference levels and power control feasibility. While at the outset, addressing the close interaction between each of these components appears challenging, we show that it is possible to come up with distributed cross layer design solutions, entailing a joint design of the physical, link, and routing layers. Further, we show that the particular design choices made here, i.e., dynamic forwarding node selection, concurrent transmissions, power control and scheduling, lead to an improved throughput per power metric for the network.

\section{System Model AND Assumptions}

We consider a wireless ad hoc network where nodes are non-uniformly distributed over a geographic area. We consider the case where "clusters" of nodes exist and that the distance between clusters is larger than the distance between nodes within a cluster. An example of such a network with two clusters is given in Fig. 1. We assume that each node generates independent traffic and attempts to forward packets to its destination whenever it has a packet to send. Further, each node can buffer packets in order to resolve conflicts.

\section{A. Physical Layer and MAC}

We assume that each node is assigned a unique signature sequence with which it will modulate its symbols. A common frequency band is used for all transmissions and several nodes can transmit simultaneously forming a CDMA ad hoc network. We assume the signature sequences are pseudo randomly generated. All necessary control information will be communicated through an additional (narrow) control channel employing TDMA, i.e., nodes take turn to transmit. We also assume that each node knows the location of each other node, and that the network topology is constant in the duration of interest. Thus, we assume any node is able to estimate its channel gain to any other node.

Each node is equipped with one antenna and nodes cannot transmit and receive at the same time. The routing policy dictates that if a node receives a packet along one sourcedestination route and at the same time must act as the transmitter for another source-destination route, then it delays its role in one of the two routes. To facilitate this scheduling, we assume a slotted structure where each slot/frame will contain concurrent transmissions.
Receiving nodes use banks of filters matched to the signatures of actively transmitting nodes. The transmission model is similar to that of [4], although, as will be evident shortly, we will not use fixed cluster heads. We note that while matched filters are suboptimum in a multiuser setting, the simplicity of the receiver structure and the use of power control to overcome near-far effects renders this particular choice a good starting point. Reference [14] proposed the use of successive interference cancellers (SIC), and no power control. However, we note that this approach requires user ordering at each receiver to yield good performance.

We assume reliable communication is possible if the QoS constraint is satisfied. We adopt signal-to-interference ratio (SIR) as the QoS; hence, the transmission from node $i$ is received reliably at node $j$ if the received SIR, $\gamma_{i j}$, is above a threshold $\gamma^{*}$. For this CDMA system with $M$ active nodes and processing gain $N$, we require:

$$
\gamma_{i j}=\frac{p_{i} h_{i j}}{\frac{1}{N} \sum_{m=1, m \neq i}^{M} p_{m} h_{m j}+\sigma^{2}} \geq \gamma^{*},
$$

where $p_{m}$ is the transmit power of node $m, h_{m j}$ is the channel gain of node $m$ at node $j$, and $\sigma^{2}$ is the variance of the additive white Gaussian channel noise. Note that the total interference experienced by the signal from node $i$ at node $j$ is

$$
I_{i j}=\frac{1}{N} \sum_{m=1, m \neq i}^{M} p_{m} h_{m j}+\sigma^{2} .
$$

Thus, if we employ power control with no maximum power constraints, where each active node transmits with just enough transmit power to achieve their SIR target $\gamma^{*}$, then up to $N / \gamma^{*}$ simultaneous transmissions can be supported. In general, power controlled CDMA ad hoc networks can easily accommodate concurrent transmissions.

\section{B. Distributed Power Control}

The iterative power control algorithm where node $i$ with intended receiver node $j$ updates its transmit power via

$$
p_{i}(n+1)=\frac{\gamma^{*}}{h_{i j}} I_{i j}(n)
$$

has been shown to converge to the unique fixed point such that node $i$ achieves its SIR target $\gamma^{*}$ [15]. This transmit power update has to be performed by all concurrently transmitting nodes in a distributed fashion since there is no base station to oversee all transmissions. The control channel can be used to facilitate this coordination. Specifically, we assume each node $j$ "measures" its received interference and then broadcasts this quantity, given as

$$
J_{j}=\frac{1}{N} \sum_{m=1}^{M} p_{m} h_{m j}+\sigma^{2} .
$$

Since each transmitting node $i$ knows its channel gain to its intended receiver $j$, it updates its power as:

$$
p_{i}(n+1)=\frac{\gamma^{*}}{h_{i j}}\left(J_{j}(n)-\frac{p_{i}(n) h_{i j}}{N}\right) .
$$


Thus, this is a completely distributed update.

\section{Motivation For Routing CHOICES}

We demonstrated above that several concurrent transmissions can be supported with power control for a CDMA ad hoc network. Next, we consider how to route information from a source to a destination so that the throughput per unit power for this transmission is maximized. In particular, we ask when it will pay-off to use multiple-hops through forwarding nodes, versus direct transmission. In this section, we present a simple analysis that yields guiding principles for constructing routing tables in clustered CDMA ad hoc networks.

Consider a network with two clusters of nodes, $C_{1}$ and $C_{2}$. Assume $C_{1}$ has at least $L+2$ nodes and $C_{2}$ has at least 1 node. Now, assume a node, say node $S$, in $C_{1}$ wishes to transmit to node $X$ in $C_{2}$. Additionally, node $A$ in $C_{1}$ wants to transmit to node $B$ in $C_{1}$. Based on this configuration, we wish to determine which of the following two routing options leads to a higher throughput per unit power:

- Case 1: Node $S$ transmits to node $X$ directly, at the same time node $A$ transmits to node $B$.

- Case 2: Node $S$ uses $L-1$ hops in $C_{1}$ to transmit to node $n$ in $C_{1}$ and node $n$, i.e., the forwarding node, transmits $S$ 's packets to node $X$ directly. When node $n$ transmits to node $X$, we assume that node $A$ simultaneously transmits to node $B$.

We assume that the channel gains are determined by path loss only, i.e., a signal transmitted at power $P_{T}$ is received at a distance $d$ with power $P_{R}=P_{T} / d^{\alpha} . \alpha$ is the path loss constant, which we assume to equal 4.

a) Case 1: Both nodes $S$ and $A$ simultaneously transmit to their desired destination nodes, i.e., nodes $X$ and $B$, respectively. The distance between nodes $S$ and $X$ is $d_{S X}$ and the distance between nodes $A$ and $B$ is $d_{A B}$. The transmit powers used are $P_{S X}$ and $P_{A B}$ at nodes $S$ and $A$, respectively. The received SIR requirements at nodes $X$ and $B$ are

$$
\gamma_{S X}=\frac{N \frac{P_{S X}}{d_{S X}^{4}}}{\frac{P_{A B}}{d_{A X}^{4}}+N \sigma^{2}} \geq \gamma^{*}, \quad \gamma_{A B}=\frac{N \frac{P_{A B}}{d_{A B}^{4}}}{\frac{P_{S X}}{d_{S B}^{4}}+N \sigma^{2}} \geq \gamma^{*}
$$

Let $K=N / \gamma^{*}+1$. We assume $d_{A X} \approx d_{S X}$ and $d_{S B} \approx d_{A B}$; then the transmit power levels $P_{S X}$ and $P_{A B}$ required to meet the SIR requirements are

$$
\begin{aligned}
P_{S X} & =\frac{N \sigma^{2}\left(d_{S X}^{4}(K-1)+d_{A B}^{4}\right)}{K-2}, \\
P_{A B} & =\frac{N \sigma^{2}\left(d_{A B}^{4}(K-1)+d_{S X}^{4}\right)}{K-2} .
\end{aligned}
$$

The total transmit power for Case 1 is therefore

$$
P_{1}=P_{S X}+P_{A B}=a\left(d_{S X}^{4}+d_{A B}^{4}\right),
$$

where $a=N \sigma^{2} /(K-2)$.

b) Case 2: Here, there will be $L-1$ single-node transmissions, i.e., transmissions from node $S$ to node $n$, and a simultaneous transmission between nodes $n$ and $X$ and nodes $A$ and $B$. Let us denote the sequence of nodes in this multihop route as $n_{1}, n_{2}, \ldots, n_{L}$, where $n_{1}=S, n_{L-1}=n, n_{L}=X$. Let $d_{i j}$ denote the distance between node $i$ and $j$. Since the $L-1$ transmissions from node $S$ to $n$ are single-node transmissions (no other interferers), it can be shown that the total power to reach node $n$ from $S$ is

$$
\frac{a(K-2)}{K-1} \sum_{i=n_{1}, n_{2}, \ldots n_{L-1}} d_{i, i+1}^{4} .
$$

During the last hop, the two simultaneous transmissions from node $n$ to $X$ and node $A$ to $B$ require total power $a\left(d_{n X}^{4}+\right.$ $\left.d_{A B}^{4}\right)$. The total power for Case 2 is therefore

$$
P_{2}=a\left(d_{n X}^{4}+d_{A B}^{4}+\frac{K-2}{K-1} \sum_{i=n_{1}, n_{2}, \ldots n_{L-1}} d_{i, i+1}^{4}\right) .
$$

Case 1 supports two source-destination pairs over a given time interval. Case 2, on the other hand, requires $L$ times longer time interval to support the same two source-destination pairs. The throughput per power for case $1, \tau_{1}$, and the throughput per power for case $2, \tau_{2}$, are given as

$$
\tau_{1}=\frac{1}{P_{1}}, \quad \tau_{2}=\frac{1}{L P_{2}} .
$$

In order to compare these two measures, $\tau_{1}$ and $\tau_{2}$, we make a simplifying assumption that the nodes within a cluster are typically separated by a distance $\frac{d_{n X}}{m}$, where $m=2,3, \ldots$. In this case, we can show that $\tau_{2}$ is greater than $\tau_{1}$ if

$$
d_{S X}>\left(L+\frac{L-1}{m^{4}}+\frac{L(L-1)(K-2)}{m^{4}(K-1)}\right)^{\frac{1}{4}} d_{n X} .
$$

This condition gives a guideline for when to use multihop transmissions. To gain some insight about this condition, consider the case of $L=2$, i.e., a two hop route from $S$ to $X$. In this case, $\tau_{2}>\tau_{1}$ if $d_{S X}>\lambda(K, m) d_{n X}$, where

$$
\lambda(K, m)=\left(\frac{2(K-2)}{m^{4}(K-1)}+\frac{1}{m^{4}}+2\right)^{\frac{1}{4}} .
$$

It can be shown that $\lambda(K, m)$ decreases as $m$ increases. Thus, the most strict requirement on the $d_{n X}$ occurs when $m=2$. Further, $\lambda(K, 2)$ increases as $K$ increases. In the limit $K \rightarrow$ $\infty, \lambda(\infty, 2)$ is 1.2161. As the distance between nodes in a cluster are assumed to be $d_{n X} / 2$, we see that for a large range of locations of node $S$, it is more beneficial to use a two-hop route via node $n$ to reach destination node $X$.

\section{NETWORK DESIGN}

The preceding analysis tells us that with careful choice of the forwarding node $n$, we can improve the energy efficiency between a source and a destination in CDMA ad hoc networks. In particular, it shows the need for multihop transmission under certain topologies. It also implies, not unexpectedly, that using the same forwarding node within a cluster for all source destination pairs is suboptimal even if groups of nodes appear to naturally form clusters. The conventional wisdom that motivates the use of cluster heads to coordinate and forward 
transmissions [4], [7], [11], [14] result in suboptimal routing policies. Given that concurrent transmissions are allowed and distributed power control is feasible, there is little need for a fixed coordinating node. Instead, we propose to dynamically choose the forwarding node for each source-destination pair, and implement a multihop scheme, only when that is the energy efficient choice.

We construct energy efficient routing tables for all source and destination pairs, identifying forwarding nodes per sourcedestination pair for both inter-cluster and intra-cluster communication, but assuming no interference. Such a routing policy is suboptimal to one that adapts to interference conditions, but will still demonstrate improvement over schemes with fixed forwarding nodes, as shown in numerical results. One alternative is to develop larger routing tables that account for various possibilities for the interference encountered by each node; this is not considered here.

When there are several source destination pairs, each with an associated route, we need to ensure no conflicts exist and that all concurrent transmissions can be supported. To do so, we implement a greedy scheduling algorithm that gives higher priority to longer routes in a distributed fashion. Specifically, at each time slot, each node identifies whether it is required to transmit and receive at the same time due to two or more routes. If this is the case, transmit/receive conflict is resolved by delaying the node's participation in the shorter route and allowing it to either transmit or receive as per the longer route. The distributed power control algorithm enables the success of each concurrent transmission in the time slot.

\section{Numerical Results}

To demonstrate the benefit of concurrent transmissions and multiple forwarding nodes, we consider a two-cluster ad hoc network with 10 nodes in each cluster. Specifically, we consider the two cluster network shown in Fig. 1.

We compare three routing strategies, and for each, we first develop the routing tables known to all nodes. The first routing table is the dynamic forwarding node based scheme in which throughput/power is maximized assuming no interfering transmissions. We then develop a routing table that follows the same principle (maximize throughput/power) for intra-cluster communication. However, when nodes wish to communicate to nodes in the second cluster, they must forward their packets to a fixed forwarding node in their own cluster. This forwarding node transmits these packets to a fixed forwarding node in the second cluster, which in turn uses maximum throughput/power routing scheme to forward packets to the destination node. In implementing the fixed forwarding mechanism, we select as forwarding nodes the two nodes in the two clusters that are closest to each other. We observed that any other selection lead to worse performance than reported here. In the third routing table, we implement the cluster head scheme. Here all source nodes transmit their packets to the cluster head. The cluster head in turn forwards the packets to the destination node, if the destination node is within the same cluster. If the destination node is in the second cluster, the cluster head transmits to a forwarding node (the same forwarding nodes as in table 2), which in turn transmits to the second cluster's cluster head. The cluster head then forwards packets to the destination node [14].

We fix the number of inter-cluster source-destination pairs and select these pairs randomly. Similarly, we fix the number of intra-cluster source-destination pairs and choose them randomly within the two clusters. In this simulation, we develop results assuming 5000 random selections of pairs.

Fig. 2 shows throughput per unit power expended versus $K=N / \gamma^{*}+1$ when one inter-cluster source-destination pair and two (one in each cluster) intra-cluster pairs communicate. The parameter $K$ relates to the maximum number of concurrent successful transmissions possible. As expected, the energy efficiency increases as the capacity increases, as the CDMA system is effectively less loaded. We observe that the routing policy where multiple dynamic forwarding nodes (MFN) are employed outperforms fixed forwarding nodes (FFN) and cluster head routing (CH). In addition, we observe that allowing concurrent transmissions for all routing policies improves the performance, demonstrating the advantage of power controlled CDMA.

Fig. 3 shows the throughput per unit power versus the number of active intra-cluster pairs when one and two intercluster pairs are communicating. The reduction in efficiency when the second inter-cluster pair joins is due to additional interference it creates which results in higher expended power for all users. Nevertheless, all users can be supported at their target SIR. In particular, the use of forwarding nodes yields higher energy efficiency with the additional inter-cluster pair as compared to the cluster head assisted routing with a single inter-cluster pair.

Finally, Fig. 4 demonstrates the energy efficiency of the multiple dynamic forwarding nodes versus the number of active intra-cluster pairs for different system capacity values, when one inter-cluster pair is also actively communicating. As expected, the throughput per unit power improves as more capacity, facilitated by higher processing gain and/or lower SIR target, is available.

\section{CONCLUSION}

In this paper, we have investigated clustered CDMA ad hoc network design while jointly addressing physical, link and network layer issues. The employment of CDMA and distributed power control enable concurrent transmissions for the system. We have investigated energy efficient routing policies, and found that dynamic forwarding nodes selected per source-destination pair outperforms policies where nodes first need to communicate to their cluster heads and policies where a fixed forwarding node is used per cluster.

We should note that in our design, we often traded simplicity for optimality, using simple receivers, a suboptimal scheduler to resolve transmit/receive conflicts and a static routing table. While even under these choices, the design benefited from the cross layer approach, and from abandoning the more structured routing policies, the energy efficiency can be further improved 


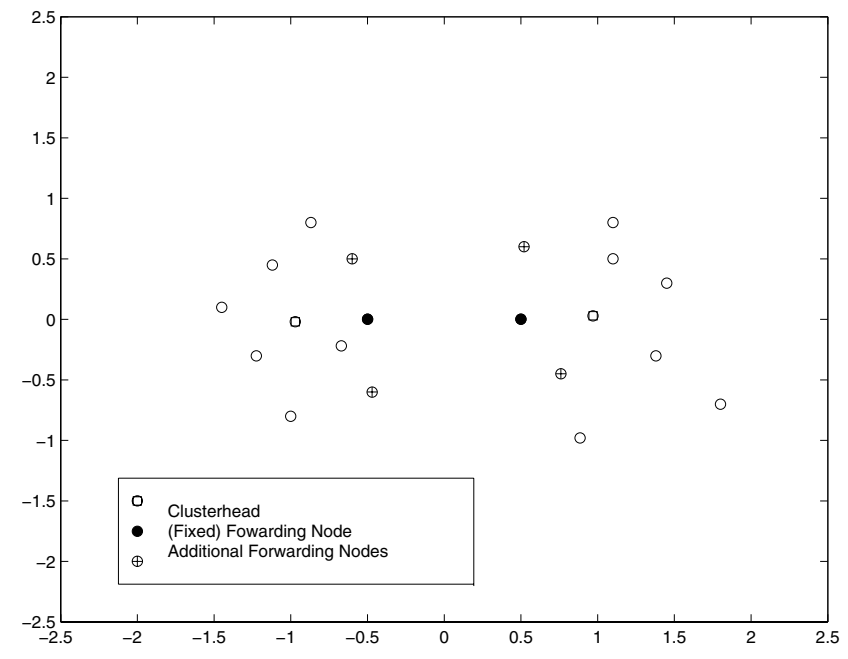

Fig. 1. The network used for the numerical results.

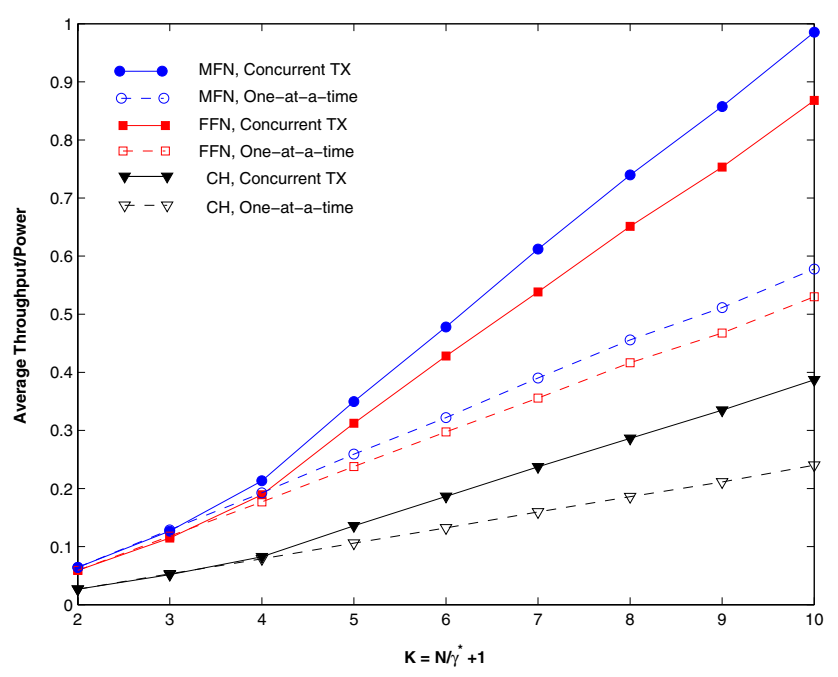

Fig. 2. Throughput/power versus capacity for one inter-cluster and two intracluster source destination pairs.

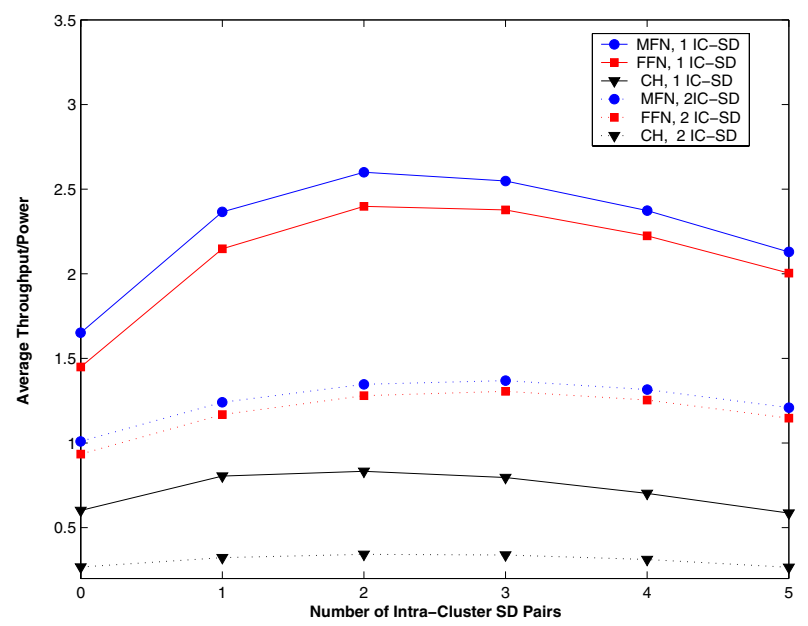

Fig. 3. Throughput/power versus number of intra-cluster source-destination pairs; $K=8$.

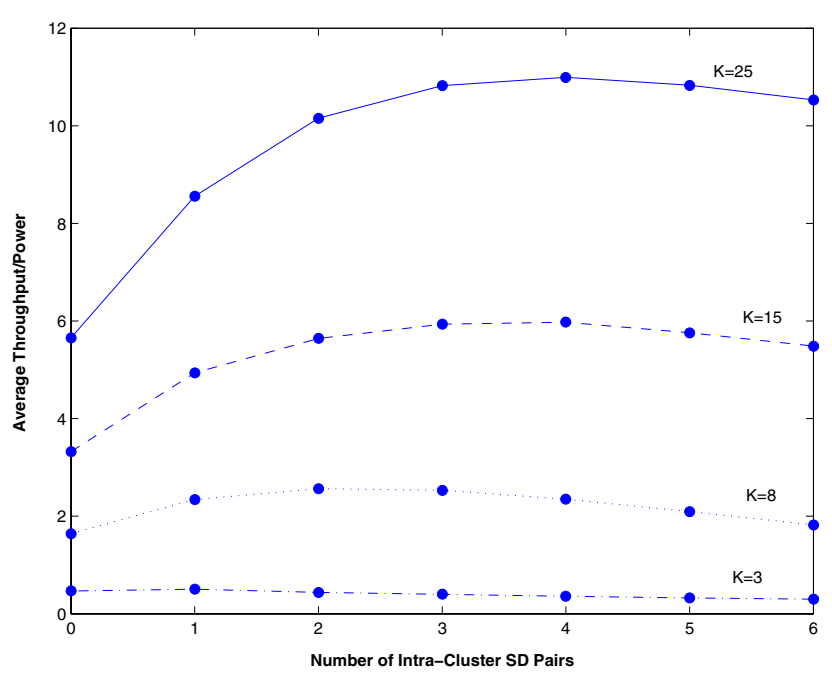

Fig. 4. Throughput/power versus number of intra-cluster source-destination pairs for routing with dynamic forwarding nodes.

with advanced receivers, schedulers, and interference-adaptive routing which a topic of current interest.

\section{REFERENCES}

[1] S. Shakkottai, T. S. Rappaport, and P. C. Karlsson. Cross-layer design for wireless networks. IEEE Comm. Magazine, Oct. 2003.

[2] W. Yuen, H. Lee, and T. Andersen. A simple and effective cross layer networking system for mobile ad hoc networks. In PIMRC, 2002.

[3] A. Muqattash and M. Krunz. CDMA-Based MAC protocol for wireless ad hoc networks. In MOBIHOC, 2003.

[4] T. ElBatt and A. Ephremides. Joint scheduling and power control for wireless ad hoc networks. IEEE Trans. on Wireless Comm., 3(1):74-85, Jan. 2004.

[5] A. Agharwal et al. Distributed power control ad-hoc wireless networks. In PIMRC, 2001.

[6] S. Wu, Y. Tseng, and J. Sheu. Intelligent medium access for mobile ad hoc networks with busy tones and power control. IEEE JSAC, 18(9):1647-1657, Sept. 2000.

[7] V. Kawaida and P. R. Kumar. Power control and clustering in ad hoc networks. In INFOCOM, 2003.

[8] J. Monks, V. Bharghavan, and W. W. Hu. A power controlled multiple access protocol for wireless packet networks. In INFOCOM, 2001.

[9] A. Behzad and I. Rubin. Power controlled multiple access control for wireless access nets. In VTC, 2003.

[10] C. R. Lin and M. Gerla. Adaptive clustering for mobile wireless networks. IEEE JSAC, 15(9):1265-1275, Sept. 1997.

[11] T. Kwon and M. Gerla. Clustering with power control. In MILCOM, 1999.

[12] D. Famolari, N. B. Mandayam, D. Goodman, and V. Shah. A new framework for power control in wireless data networks: Games, utility and pricing. In Wireless Multimedia Network Technologies, pp. 289-310. Kluwer Academic Publishers, 1999.

[13] A. Chockalingam et al. Energy efficiency analysis of a multichannel wireless access protocol. In PIMRC, 1998.

[14] A. Hasan, K. Yang, and J. G. Andrews. Clustered CDMA ad hoc networks without closed-loop power control. In MILCOM, 2003.

[15] R. D. Yates. A framework for uplink power control in cellular radio systems. IEEE JSAC, 13(7):1341-1347, Sept. 1995. 